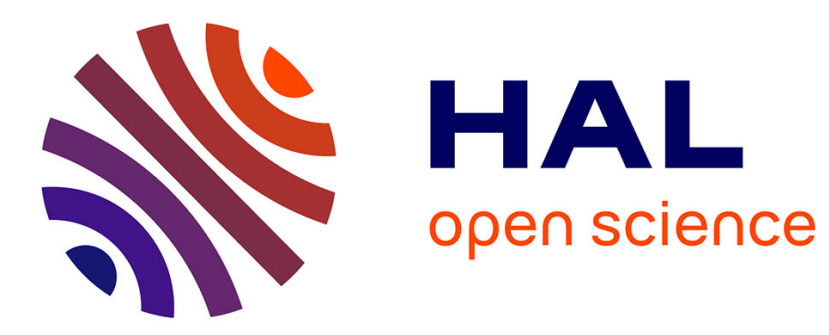

\title{
Replica-Mean-Field Limits of Fragmentation-Interaction-Aggregation Processes
}

François Baccelli, Michel Davydov, Thibaud Taillefumier

\section{To cite this version:}

François Baccelli, Michel Davydov, Thibaud Taillefumier. Replica-Mean-Field Limits of Fragmentation-Interaction-Aggregation Processes. Journal of Applied Probability, 2022, 59 (1), pp.3859. 10.1017/jpr.2021.31 . hal-02607064

\section{HAL Id: hal-02607064 https://hal.science/hal-02607064}

Submitted on 16 May 2020

HAL is a multi-disciplinary open access archive for the deposit and dissemination of scientific research documents, whether they are published or not. The documents may come from teaching and research institutions in France or abroad, or from public or private research centers.
L'archive ouverte pluridisciplinaire HAL, est destinée au dépôt et à la diffusion de documents scientifiques de niveau recherche, publiés ou non, émanant des établissements d'enseignement et de recherche français ou étrangers, des laboratoires publics ou privés. 


\title{
Replica-Mean-Field Limits of \\ Fragmentation-Interaction-Aggregation Processes
}

\author{
François Baccelli *
}

Michel Davydov ${ }^{\dagger}$

Thibaud Taillefumier $\ddagger$

\begin{abstract}
Network dynamics with point-process-based interactions are of paramount modeling interest. Unfortunately, most relevant dynamics involve complex graphs of interactions for which an exact computational treatment is impossible. To circumvent this difficulty, the replica-mean-field approach focuses on randomly interacting replicas of the networks of interest. In the limit of an infinite number of replicas, these networks become analytically tractable under the so-called "Poisson Hypothesis". However, in most applications, this hypothesis is only conjectured. Here, we establish the Poisson Hypothesis for a general class of discrete-time, point-process-based dynamics, that we propose to call fragmentationinteraction-aggregation processes, and which are introduced in the present paper. These processes feature a network of nodes, each endowed with a state governing their random activation. Each activation triggers the fragmentation of the activated node state and the transmission of interaction signals to downstream nodes. In turn, the signals received by nodes are aggregated to their state. Our main contribution is a proof of the Poisson Hypothesis for the replica-mean-field version of any network in this class. The proof is obtained by establishing the propagation of asymptotic independence for state variables in the limit of an infinite number of replicas. Discrete time Galves-Löcherbach neural networks are used as a basic instance and illustration of our analysis.
\end{abstract}

\section{Introduction}

Epidemics propagation, chemical reactions, opinion dynamics, flow control in the Internet, and even neural computations can all be modelled via punctuate interactions between interconnected agents [15][11][1][2][17]. The phenomena of interest in this context are idealized as network dynamics on a graph of agents which interact via point processes: edges between agents are the support of interactions, with edge-specific point processes registering the times at which these interactions are exerted. Such point-process-based network dynamics constitute a very versatile class of models able to capture phenomena in natural sciences, engineering, social sciences and economics.

However, this versatility comes at the cost of tractability as the mathematical analysis of these dynamics is impossible except in the simplest network architectures. As a result, to go beyond numerical simulations, one has to resort to simplifying assumptions.

\footnotetext{
*Département d'informatique de l'ENS, ENS, CNRS, PSL University, Paris, France

†Département d'informatique de l'ENS, ENS, CNRS, PSL University, Paris, France

$\ddagger$ Department of Mathematics and Department of Neuroscience, University of Texas, Austin, TX
} 
For such point-process-based network dynamics, the main obstacle to computational tractability is the fact that the interaction point processes arising in the dynamics do not belong to any parametric class. Replica Mean-Fields (RMF) are meant to get around this obstacle. The RMF version of a given network is an extension of this network built in such a way that interaction point processes are parametric, e.g., Poisson. This extended network is made of infinitely many replicas of the initial network, all with the same basic structure, but with randomized interactions across replicas. The interest in RMFs stems from the fact that they offer tractable version of the original dynamics that retain some of its most important features. The fact that Poisson point processes arise in the RMF version of a network is called the Poisson Hypothesis. Thus formulated, the Poisson Hypothesis originates from communication network theory [12] and is distinct from replica approaches developed in statistical physics [6].

Although intuitively clear and despite its usefulness, the Poisson Hypothesis is often only conjectured and/or numerically validated. This is for instance the case in recent work on neural network dynamics [3]. The purpose of this work is to rigorously establish the Poisson Hypothesis for the RMF limits of a broad class of discrete-time point process based network dynamics introduced in the present paper. This class, which will be referred to as fragmentation-interaction-aggregation processes (FIAPs) below, includes discrete time Galves-Löcherbach neural networks as a special case.

\section{Fragmentation-interaction-aggregation processes}

In FIAPs, agents are graph nodes endowed with a state that evolves over time. The nodes are coupled via point processes which model punctuate interactions. Specifically, each node's state evolves in response to its input point process, and generates an output point process in a state-dependent manner. In all generality, the transformation of input into output point process can be viewed as a random map. In FIAPs, this map is defined through the following dynamics:

(i) The fragmentation process is triggered by local activation events taking place on each node and which occur with a probability that depends on the state of the node.

(ii) Each fragmentation event in turn triggers interactions between the nodes by creating input events in the neighboring nodes.

(iii) Finally, the aggregation process consists in the integration of the input point processes to the states of each node.

Thus broadly defined, FIAPs offer a simple albeit general framework to analyze the phenomena alluded to above. The precise definition of FIAPs is given as follows:

Definition 1. An instance of the class $\mathcal{C}$ of discrete fragmentation-interaction-aggregation processes is determined by:

- An integer $K$ representing the number of nodes;

- A collection of initial conditions for the integer-valued state variables at step zero, which we denote by $\left\{X_{i}\right\}$, where $i \in\{1, \ldots, K\}$;

- A collection of fragmentation random variables $\left\{U_{i}\right\}$, which are i.i.d. uniform in $[0,1]$ and independent from $\left\{X_{i}\right\}$, where $i \in\{1, \ldots, K\}$;

- A collection of fragmentation functions $\left\{g_{1, i}: \mathbb{N} \rightarrow \mathbb{N}\right\}_{i \in\{1, \ldots, K\}}$ and $\left\{g_{2, i}: \mathbb{N} \rightarrow \mathbb{N}\right\}_{i \in\{1, \ldots, K\}}$;

- A collection of bounded interaction functions $\left\{h_{i, j}: \mathbb{N} \rightarrow \mathbb{N}\right\}_{i, j \in\{1, \ldots, K\}}$;

- A collection of activation probabilities $\left\{\sigma_{i}(0), \sigma_{i}(1), \ldots\right\}_{i \in\{1, \ldots, K\}}$ verifying the conditions $\sigma_{i}(0)=0$, and $0<\sigma_{i}(1) \leq \sigma_{i}(2) \leq \cdots \leq 1$ for all $i$. 
The associated dynamics take as input the initial integer-valued state variables $\left\{X_{i}\right\}$ and define the state variables at the next step as

$$
Y_{i}=g_{1, i}\left(X_{i}\right) \mathbb{1}_{\left\{U_{i}<\sigma_{i}\left(X_{i}\right)\right\}}+g_{2, i}\left(X_{i}\right) \mathbb{1}_{\left\{U_{i}>\sigma_{i}\left(X_{i}\right)\right\}}+A_{i}, \quad \forall i=1, \ldots, K,
$$

with arrival processes

$$
A_{i}=\sum_{j \neq i} h_{i, j}\left(X_{j}\right) \mathbb{I}_{\left\{U_{j}<\sigma_{j}\left(X_{j}\right)\right\}}, \quad \forall i=1, \ldots, K .
$$

The interpretation is as follows: node $i$ activates with probability $\sigma_{i}(k)$ if its state $X_{i}$ is equal to $k$. The state of this node is fragmented to $g_{1, i}(k)$ upon activation and to $g_{2, i}(k)$ otherwise. This activation triggers an input of $h_{j, i}(k)$ units to node $j$. Hence, the interaction functions encode the structure of the graph. The variable $A_{i}$ gives the total number of arrivals to node $i$. This variable is aggregated to the state of the node as seen in (1). Note that considering $\sigma_{i}(0)=0$ for all $i$ ensures that the state variables remain non-negative.

Thus precisely defined, the FIAP class $\mathcal{C}$ encompasses many network dynamics relevant to queuing theory and mathematical biology. For example, taking $g_{1, i}(k)=k-1, g_{2, i}(k)=k$ and $\left.h_{i, j}(k)=\mathbb{I}_{\{i=j+1} \bmod K\right\}$, we recover an instance of Gordon-Newell queuing networks [13]. Taking $g_{1, i}(k)=0, g_{2, i}(k)=k$ and $h_{i, j}(k)=\mu_{i, j} \in \mathbb{N}$ defines a discrete instance of linear Galves-Löcherbach dynamics for neural networks (see next section). Taking $g_{1, i}(k)=\left\lfloor\frac{k}{2}\right\rfloor$ and $g_{2, i}(k)=k+1$, corresponds to aggregation-fragmentation processes modelling, e..g., cellular growth [8] or TCP communication networks [2]. Note that the class $\mathcal{C}$ also includes certain discrete time Hawkes processes. Namely, if for each coordinate of a Hawkes vector process, we define its state as the sum over time of all its variations, then all discrete Hawkes processes that are Markov with respect to their so-defined state are in $\mathcal{C}$.

The present paper is focused on discrete time versions of this type of dynamics as in, e.g., [16] [7]; note that continuous versions were also considered in the literature such as in [8], [14].

\section{Replica models for fragmentation-interaction-aggregation networks}

Finite RMF models are defined as a coupling of replicas of the network of interest by randomized routing decisions. For a FIAP, the state of its $M$-replica model is thus specified by a collection of state variables $X_{m, i}^{M}$, where $m$ is the index of the replica and $i$ corresponds to the index of the node in the original network. Instead of interacting with nodes within the same replica, an activated node $i$ in replica $m$ interacts with a downstream node $j$ from a replica $m$ chosen uniformly at random and independently. This randomization preserves essential features of the original dynamics such as the magnitude of interactions between nodes but degrades the dependence structure between nodes. Indeed, over a finite period of time, the probability for a particular node to receive an activation from another given node scales as $1 / M$. Thus, as the number of replicas increases, interactions between distinct replicas become ever scarcer, intuitively leading to replica independence when $M \rightarrow \infty$. This asymptotic independence is the root of RMF computational tractability.

Here is the precise definition of the finite-replica version of a FIAP:

Definition 2. For any process in $\mathcal{C}$, the associated $M$-replica dynamics is entirely specified by

- A collection of initial conditions for the integer-valued state variables at step zero, which we denote by $\left\{X_{n, i}^{M}\right\}$, where $n \in\{1, \ldots, M\}$ and $i \in\{1, \ldots, K\}$, such that for all $M, n$ and $i, X_{n, i}^{M}=X_{i}$;

- A collection of fragmentation random variables $\left\{U_{n, i}\right\}$, which are i.i.d. uniform in $[0,1]$ and independent from $\left\{X_{n, i}^{M}\right\}$, where $n \in\{1, \ldots, M\}$ and $i \in\{1, \ldots, K\}$;

- A collection of i.i.d. routing random variables $\left\{R_{m, j, i}^{M}\right\}$ independent from $\left\{X_{n, i}^{M}\right\}$ and $\left\{U_{n, i}\right\}$, uniformly distributed on $\{1, \ldots, M\} \backslash\{m\}$ for all $i, j \in\{1, \ldots, K\}$ and $m \in\{1, \ldots, M\}$. In other words, if $R_{m, j, i}^{M}=n$, then an eventual activation of node $j$ in replica $m$ at step 0 induces an arrival of size 
$h_{i, j}\left(X_{m, j}^{M}\right)$ in node $i$ of replica $n$, and $n$ is chosen uniformly among replicas and independently from the state variables. Note that these variables are defined regardless of the fact that an activation actually occurs. Also note that for $i^{\prime} \neq i$, the activation in question will induce an arrival in node $i^{\prime}$ of replica $n^{\prime}$, with $n^{\prime}$ sampled in the same way but independently of $n$.

Then, the integer-valued state variables at step one, denoted by $\left\{Y_{n, i}^{M}\right\}$, are given by the $M$-RMF equations

$$
Y_{n, i}^{M}=g_{1, i}\left(X_{n, i}^{M}\right) \mathbb{I}_{\left\{U_{n, i}<\sigma_{i}\left(X_{n, i}^{M}\right)\right\}}+g_{2, i}\left(X_{n, i}^{M}\right) \mathbb{I}_{\left\{U_{n, i}>\sigma_{i}\left(X_{n, i}^{M}\right)\right\}}+A_{n, i}^{M},
$$

where $g_{1, i}, g_{2, i}$ denotes fragmentation functions, $\sigma_{i}$ denotes activation probabilities, and where

$$
A_{n, i}^{M}=\sum_{m \neq n} \sum_{j \neq i} h_{i, j}\left(X_{m, j}^{M}\right) \mathbb{1}_{\left\{U_{m, j}<\sigma_{i}\left(X_{m, j}^{M}\right)\right\}} \mathbb{1}_{\left\{R_{m, j, i}^{M}=n\right\}}
$$

is the number of arrivals to node $i$ of replica $n$ via the interaction functions $h_{i, j}$.

RMF models are only expected to become tractable when individual replicas become independent. This happens in the limit of an infinite number of replicas, i.e., in the so-called RMF limit [3]. In this RMF limit, asymptotic independence between replicas follows from the more specific Poisson Hypothesis. The Poisson Hypothesis states that spiking deliveries to distinct replicas shall be asymptotically distributed as independent Poisson (or compound) point processes. Such a hypothesis, which has been numerically validated for certain RMF networks, has been conjectured for linear Galves-Löcherbach dynamics in [3]. Proving the validity of the Poisson Hypothesis for the RMF limits of the much more general FIAPs is the purpose of the present work.

\section{Methodology for proving the Poisson Hypothesis}

Classical mean-field approximations of a given network are obtained by considering the limit of the original network when a certain characteristic of the network - typically the number of nodes - goes to infinity. When the dynamics of the nodes are synchronous, one gets a discrete time dynamical system. The term mean-field comes from the fact that in such network limits, the effect that individual nodes have on one another are approximated by a single averaged effect, typically an empirical mean. In the limit, this empirical mean usually converges to an expectation term through a propagation of chaos result [18] which leads to analytical tractability. In replica mean-fields, there is no such empirical mean over the nodes of the network; the mean-field simplification comes from the random routing operations between replicas. The input point process in the $M$-replica model consists in a superposition of $M$ rare point processes, which informally explains why Poisson (or compound Poisson) processes arise at the limit. For classical mean-fields, different techniques have been developed to prove the existence and the convergence to the mean-field limit. Standard techniques include the use of the theory of nonlinear Markov processes [19], stochastic approximation algorithms[4], and induction techniques which assume the existence of limits at time zero and extend the result by induction [5]. Refinements to the latter approach can be made in order to obtain explicit rates of convergence [10]. The approach developed for the RMF case belongs in spirit to the third class of techniques. We suppose that the property of asymptotic independence (see Definition 3) holds for the state variables at time zero. We then prove that this property is preserved by the dynamics of the $M$-replica model and thus holds by induction for any finite time. We show that this asymptotic independence hypothesis implies both convergence in distribution and an ergodic type property that we call the triangular law of large numbers. We apply this law of large numbers to the input process to a single node to show that Poisson (or compound Poisson) processes appear in the replica mean-field limit indeed. 


\section{Structure of the Paper}

For the sake of clarity in exposition, we start with the proof of the Poisson Hypothesis for the special case of neural networks first before extending it to general FIAPs. More precisely, we first consider the symmetric neural network case, which is a fully symmetric Galves-Löcherbach model [9] in discrete time. We introduce the model in Section 2 and prove the Poisson Hypothesis in Section 3. We then extend the proof to the class of FIAPs defined above. We first consider the symmetric case in Section 4 and then the general case in Section 5. Finally, some extensions are discussed in Section 6.

\section{The symmetric Galves-Löcherbach model}

\subsection{The symmetric RMF network model}

We consider a network of $K$ spiking neurons. We suppose that the behavior of each neuron is determined by a random variable representing the membrane potential of the neuron. Each neuron spikes at a rate depending on its state variable. Let $X=\left\{X_{i}\right\}$ be the integer-valued state variables at step 0 , where $i \in\{1, \ldots, K\}$. Let $Y=\left\{Y_{i}\right\}$ be the integer-valued state variables at time one. The system continues to evolve in discrete time with all corresponding state variables defined by induction.

Let $\sigma: \mathbb{N} \rightarrow[0,1]$ be the spiking probabilities of the neurons. Namely, $\sigma(k)$ is the probability that a neuron in state $k$ spikes. We consider that $\sigma(0)=0$, accounting for the fact that a neuron in state 0 never spikes. We also consider that $\sigma(1)>0$ and that $\sigma$ is non-decreasing. Let $\left\{U_{i}\right\}$ be uniformly distributed i.i.d. random variables independent from $\left\{X_{i}\right\}$. We then write the following evolution equation for the state of the system:

$$
Y_{i}=\mathbb{I}_{\left\{U_{i}>\sigma\left(X_{i}\right)\right\}} X_{i}+A_{i}
$$

where

$$
A_{i}=\sum_{j \neq i} \mathbb{I}_{\left\{U_{j}<\sigma\left(X_{j}\right)\right\}}
$$

is the number of arrivals to neuron $i$.

Here, the fragmentation is complete if $U_{i}<\sigma\left(X_{i}\right)$, namely if there is a spike, in which case the state variable is reset (jumps to 0 ). Otherwise there is no fragmentation at all and the state variable is left unchanged. In both cases, the arrivals $A_{i}$ are aggregated to the state.

The RMF model described below is a discrete time version of the model introduced in [3]. Namely, we consider a collection of $M$ identically distributed replicas of the initial set of $K$ neurons. Let $X=\left\{X_{n, i}^{M}\right\}$ be the integer-valued state variables at step 0 , where $n \in\{1, \ldots, M\}, i \in\{1, \ldots, K\}$. Let $Y=\left\{Y_{n, i}^{M}\right\}$ be the integer-valued state variables at time one. Let $U=\left\{U_{n, i}\right\}$ be uniformly i.i.d. random variables in $[0,1]$ independent from $\left\{X_{n, i}^{M}\right\}$. Let $R=\left\{R_{m, i, j}^{M}\right\}$ be i.i.d. routing random variables independent from $\left\{X_{n, i}^{M}\right\}$ and $\left\{U_{n, i}\right\}$, uniformly distributed on $\{1, \ldots, M\} \backslash\{m\}$ for all $i, j \in\{1, \ldots, K\}$ and $m \in\{1, \ldots, M\}$. The replica model has the following evolution equation:

$$
Y_{n, i}^{M}=\mathbb{1}_{\left\{U_{n, i}>\sigma\left(X_{n, i}^{M}\right)\right\}} X_{n, i}^{M}+A_{n, i}^{M}
$$

where

$$
A_{n, i}^{M}=\sum_{m \neq n} \sum_{j \neq i} \mathbb{I}_{\left\{U_{m, j}<\sigma\left(X_{m, j}^{M}\right)\right\}} \mathbb{I}_{\left\{R_{m, j, i}^{M}=n\right\}}
$$

is the number of arrivals to neuron $i$ of replica $n$.

\subsection{Pairwise asymptotic independence and consequences}

Our goal is to show the propagation of chaos and the Poisson Hypothesis in this system. In other words, we want to show that the arrivals to two distinct replicas are asymptotically independent and the number of 
arrivals to one replica is asymptotically Poisson distributed. We begin by considering the fully exchangeable case with equal weights, but we will consider the general case later. In order to do so, we choose to characterize the propagation of chaos through the following properties:

Definition 3. Given $M \in \mathbb{N}$, given an array of integer-valued random variables $Z=\left\{Z_{n, i}^{M}\right\}_{1 \leq n \leq M, 1 \leq i \leq K}$ such that for all fixed $M$, the random variables $Z_{n, i}^{M}$ are exchangeable in $n$ and $i$, we say that the variables $Z_{n, i}^{M}$ are pairwise asymptotically independent, which we will denote $\operatorname{PAI}(Z)$, if there exists an integer-valued random variable $\tilde{Z}$ such that $\forall(n, i) \neq(m, j), \forall u, v \in[0,1]$,

$$
\lim _{M \rightarrow \infty} \mathbf{E}\left[u^{Z_{n, i}^{M}} v^{Z_{m, j}^{M}}\right]=\mathbf{E}\left[u^{\tilde{Z}}\right] \mathbf{E}\left[v^{\tilde{Z}}\right] .
$$

Definition 4. Given $M \in \mathbb{N}$, given an array of integer-valued random variables $Z=\left\{Z_{n}^{M}\right\}_{n \in\{1, \ldots, M\}}$ such that for all fixed $M$, the random variables $Z_{n}^{M}$ are exchangeable in $n$, we say that $Z$ verifies the triangular law of large numbers $\operatorname{TLLN}(Z)$ if there exists an integer-valued random variable $\tilde{Z}$ such that for all functions $f: \mathbb{N} \rightarrow \mathbb{R}$ with compact support, we have the following limit in $L^{2}$ :

$$
\lim _{M \rightarrow \infty} \frac{1}{M} \sum_{n=1}^{M} f\left(Z_{n}^{M}\right)=\mathbf{E}[f(\tilde{Z})] .
$$

Here are a few remarks about these definitions. First, note that if an array of random variables $Z$ satisfies $\operatorname{PAI}(Z)$, then for all $n$ and $i, Z_{n, i}^{M}$ converges in distribution to $\tilde{Z}$ as $M \rightarrow \infty$. This can be seen by taking $v=1$ in the definition. By considering the case where $Z_{n}^{M}=Z_{1}^{1}$ for all $n$ and $M$, we see that the convergence in distribution of $Z_{n}^{M}$ does not imply TLLN $(Z)$. However, we show below that for all arrays of random variables $Z=\left\{Z_{n, i}^{M}\right\}_{n \in\{1, \ldots, M\}, i \in\{1, \ldots, K\}}$ satisfying $\operatorname{PAI}(Z)$, for all $i, Z_{i}=\left\{Z_{n, i}^{M}\right\}_{n \in\{1, \ldots, M\}}$ satisfies $\operatorname{TLLN}\left(Z_{i}\right)$. In other words, pairwise asymptotic independence of an array of random variables implies that these random variables verify the triangular law of large numbers. The following characterization of $L^{2}$ convergence will be used throughout this paper:

Lemma 5. Let $\left(X_{n}\right)$ be random variables with finite second moments. Then there exists a constant $c$ such that $X_{n} \rightarrow c$ in $L^{2}$ when $n \rightarrow \infty$ iff

(i) $\mathbf{E}\left[X_{n}\right] \rightarrow c$ when $n \rightarrow \infty$

(ii) $\operatorname{Var}\left(X_{n}\right) \rightarrow 0$ when $n \rightarrow \infty$.

This follows directly from the definition of $L^{2}$ convergence.

The following lemma describes the relation between pairwise asymptotic independence and the triangular law of large numbers.

Lemma 6. Let $M \in \mathbb{N}$, let $Z=\left\{Z_{n, i}^{M}\right\}_{n \in\{1, \ldots, M\}, i \in\{1, \ldots, K\}}$ be an array of integer valued random variables verifying $\operatorname{PAI}(Z)$. Then, for all $i, Z_{i}=\left\{Z_{n, i}^{M}\right\}_{n \in\{1, \ldots, M\}}$ satisfies $\operatorname{TLLN}\left(Z_{i}\right)$. In other words, for all functions $f: \mathbb{N} \rightarrow \mathbb{R}$ with compact support, for all $i \in\{1, \ldots, K\}$, we have

$$
\frac{1}{M} \sum_{n=1}^{M} f\left(Z_{n, i}^{M}\right) \rightarrow \mathbf{E}[f(\tilde{Z})]
$$

in $L^{2}$ when $M \rightarrow \infty$.

Proof. Let $f: \mathbb{N} \rightarrow \mathbb{R}$ be a function with compact support. We use Lemma 5 . We fix $i \in\{1, \ldots, K\}$ that we omit in the rest of the proof. We have

$$
\begin{aligned}
\operatorname{Var}\left(\frac{1}{M} \sum_{n=1}^{M} f\left(Z_{n}^{M}\right)\right) & =\frac{1}{M^{2}}\left(\sum_{n=1}^{M} \operatorname{Var}\left(f\left(Z_{n}^{M}\right)\right)+\sum_{p \neq q} \operatorname{cov}\left[f\left(Z_{p}^{M}\right), f\left(Z_{q}^{M}\right)\right]\right) \\
& =\frac{1}{M} \operatorname{Var}\left(f\left(Z_{1}^{M}\right)\right)+\frac{M(M-1)}{M^{2}} \operatorname{cov}\left[f\left(Z_{1}^{M}\right), f\left(Z_{2}^{M}\right)\right],
\end{aligned}
$$


the last equality holding by exchangeability between replicas. Both terms on the right hand side go to 0 when $M \rightarrow \infty$. For the first term, this follows from the boundedness of $f$. For the second, we first show the result for indicator functions. Let $B \in \mathcal{B}(\mathbb{R})$ and let $f$ be defined by $f(n)=\mathbb{I}_{\{n \in B\}}$. Then we have

$$
\operatorname{cov}\left[f\left(Z_{1}^{M}\right), f\left(Z_{2}^{M}\right)\right]=\mathbf{P}\left(Z_{1}^{M} \in B, Z_{2}^{M} \in B\right)-\mathbf{P}\left(Z_{1}^{M} \in B\right) \mathbf{P}\left(Z_{2}^{M} \in B\right),
$$

which goes to 0 when $M \rightarrow \infty$ by $\operatorname{PAI}(Z)$. This immediately extends to functions with compact support since they only take a finite number of values. Moreover, for all such functions, $\mathbf{E}\left[\frac{1}{M} \sum_{n=1}^{M} f\left(Z_{n}^{M}\right)\right] \rightarrow$ $\mathbf{E}[f(\tilde{Z})]$ when $M \rightarrow \infty$ as a direct consequence of the fact that for integer-valued random variables, convergence in distribution of $Z^{M}$ to $\tilde{Z}$ is equivalent to the convergence $\mathbf{P}\left(Z^{M}=k\right) \rightarrow \mathbf{P}(\tilde{Z}=k)$ for all $k \in \mathbb{N}$. This concludes the proof.

For our subsequent needs, we also establish the following result: we show that pairwise asymptotic independence implies a property that is slightly more general than the triangular law of large numbers, where we allow the function $f$ to depend on an array of i.i.d. random variables $U=\left\{U_{n, i}\right\}_{n \in\{1, \ldots, M\}, i \in\{1, \ldots, K\}}$, independent from the rest of the dynamics.

Lemma 7 (Generalized TLLN). Let $M \in \mathbb{N}$, let $Z=\left\{Z_{n, i}^{M}\right\}_{n \in\{1, \ldots, M\}, i \in\{1, \ldots, K\}}$ be an array of integer valued random variables verifying $\operatorname{PAI}(Z)$. Then for all bounded functions $f: \mathbb{N} \times[0,1] \rightarrow \mathbb{R}$ with compact support, for all i.i.d. sequences of random variables $U=\left\{U_{n, i}\right\}_{n \in\{1, \ldots, M\}, i \in\{1, \ldots, K\}}$ independent from $Z$, there exists $\tilde{U}$ independent from $\tilde{Z}$ and $Z$ such that, for all $i \in\{1, \ldots, K\}$, we have the following limit in $L^{2}$ :

$$
\lim _{M \rightarrow \infty} \frac{1}{M} \sum_{n=1}^{M} f\left(Z_{n, i}^{M}, U_{n, i}\right)=\mathbf{E}[f(\tilde{Z}, \tilde{U})] .
$$

Note that compared to Definition 4, we consider that the functions are bounded, a condition that was automatically fulfilled for functions with compact support on $\mathbb{N}$.

Proof. We proceed as in the last lemma, conditioning on the $U_{n, i}$ when necessary. Let $M \in \mathbb{N}$, let $i \in\{1, \ldots, K\}$. We will omit this index in the rest of the proof. By exchangeability between replicas, we have

$$
\begin{aligned}
\mathbf{E}\left[\frac{1}{M} \sum_{n=1}^{M} f\left(Z_{n}^{M}, U_{n}\right)\right] & =\mathbf{E}\left[f\left(Z_{1}^{M}, U_{1}\right)\right] \\
& =\mathbf{E}\left[f\left(Z_{1}^{M}, \tilde{U}\right)\right] \text { defining } \tilde{U}=U_{1} \\
& =\mathbf{E}\left[\mathbf{E}\left[f\left(Z_{1}^{M}, \tilde{U}\right) \mid \tilde{U}\right]\right] .
\end{aligned}
$$

Since $Z_{1}^{M}$ converges in distribution to $\tilde{Z}$ when $M \rightarrow \infty$, and since $Z_{1}^{M}$ is integer-valued and $f$ is bounded, for all $u \in[0,1], \mathbf{E}\left[f\left(Z_{1}^{M}, u\right)\right] \rightarrow \mathbf{E}[f(\tilde{Z}, u)]$ when $M \rightarrow \infty$. Therefore, since $\tilde{U}$ is independent from $Z$ and $\tilde{Z}$, for all $\left.u \in[0,1], \mathbf{E}\left[f\left(Z_{1}^{M}, u\right) \mid \tilde{U}=u\right]\right] \rightarrow \mathbf{E}[f(\tilde{Z}, u) \mid \tilde{U}=u]$ when $M \rightarrow \infty$. Hence, we have that when $M \rightarrow \infty, \mathbf{E}\left[f\left(Z_{1}^{M}, \tilde{U}\right) \mid \tilde{U}\right] \rightarrow \mathbf{E}[f(\tilde{Z}, \tilde{U}) \mid \tilde{U}]$ a.s.. Finally, by the bounded convergence theorem, $\mathbf{E}\left[\mathbf{E}\left[f\left(Z_{1}^{M}, \tilde{U}\right) \mid \tilde{U}\right]\right] \rightarrow \mathbf{E}[\mathbf{E}[f(\tilde{Z}, \tilde{U}) \mid \tilde{U}]]$ when $M \rightarrow \infty$. Thus,

$$
\mathbf{E}\left[\frac{1}{M} \sum_{n=1}^{M} f\left(Z_{n}^{M}, U_{n}\right)\right] \rightarrow \mathbf{E}[f(\tilde{Z}, \tilde{U})]
$$

when $M \rightarrow \infty$.

Moreover,

$$
\begin{aligned}
\operatorname{Var}\left(\frac{1}{M} \sum_{n=1}^{M} f\left(Z_{n}^{M}, U_{n}\right)\right) & =\frac{1}{M^{2}} \sum_{n=1}^{M} \operatorname{Var}\left(f\left(Z_{n}^{M}, U_{n}\right)\right)+\frac{1}{M^{2}} \sum_{n \neq n^{\prime}} \operatorname{cov}\left[f\left(Z_{n}^{M}, U_{n}\right), f\left(Z_{n^{\prime}}^{M}, U_{n^{\prime}}\right)\right] \\
& =\frac{1}{M} \operatorname{Var}\left(f\left(Z_{1}^{M}, U_{1}\right)\right)+\frac{M(M-1)}{M^{2}} \operatorname{cov}\left[f\left(Z_{1}^{M}, U_{1}\right), f\left(Z_{2}^{M}, U_{2}\right)\right]
\end{aligned}
$$


the last equality stemming from exchangeability between replicas. When $M \rightarrow \infty$, the first term goes to 0 because $f$ is bounded. For the second term, since the $\left\{Z_{n}^{M}\right\}$ and the $\left\{U_{n}\right\}$ are independent and the $\left\{U_{n}\right\}$ are i.i.d., we can proceed as above. Namely, let $B, C \in \mathcal{B}(\mathbb{R})$. Let $f$ be defined by $f(n, t)=\mathbb{I}_{\{n \in B\}} \mathbb{I}_{\{t \in C\}}$. Then we have

$$
\begin{aligned}
\operatorname{cov}\left[f\left(Z_{1}^{M}, U_{1}\right), f\left(Z_{2}^{M}, U_{2}\right)\right]= & \mathbf{P}\left(Z_{1}^{M} \in B, Z_{2}^{M} \in B, U_{1} \in C, U_{2} \in C\right) \\
& -\mathbf{P}\left(Z_{1}^{M} \in B, U_{1} \in C\right) \mathbf{P}\left(Z_{2}^{M} \in B, U_{2} \in C\right) \\
= & \left(\mathbf{P}\left(Z_{1}^{M} \in B, Z_{2}^{M} \in B\right)-\mathbf{P}\left(Z_{1}^{M} \in B\right) \mathbf{P}\left(Z_{2}^{M} \in B\right)\right) \mathbf{P}\left(U_{1} \in C\right) \mathbf{P}\left(U_{2} \in C\right),
\end{aligned}
$$

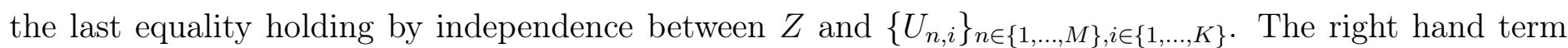
goes to 0 when $M \rightarrow \infty$ by $\operatorname{PAI}(Z)$. This generalizes to bounded functions with compact support, which concludes the proof.

\subsection{Main result}

Our goal is to show that if $X=\left\{X_{n, i}^{M}\right\}$ are asymptotically independent, then $Y=\left\{Y_{n, i}^{M}\right\}$ are as well. In other words, if we choose initial conditions that verify a certain property, this property will hold by induction at any finite discrete time.

Theorem 8. Let $M \in \mathbb{N}$, let $X=\left\{X_{n, i}^{M}\right\}_{n \in\{1, \ldots, M\}, i \in\{1, \ldots, K\}}$ be an array of integer valued random variables (the "state variables"). Suppose that $\operatorname{PAI}(X)$ holds. Then $\operatorname{PAI}(Y)$ holds as well, where $Y$ is defined by (7). Moreover, the arrivals to a given node $A_{n, i}^{M}$ converge in distribution to a Poisson random variable when $M \rightarrow \infty$.

Note that the result depends on a choice of initial conditions verifying $\operatorname{PAI}(X)$, a typical example of which is i.i.d. initial conditions stable in law, in the sense that their law does not depend on $M$. The question of whether given an arbitrary initial condition, the dynamics become pairwise asymptotically independent after some (finite or infinite) amount of time, is still open. Note also that this shows that we have convergence in distribution of the exchangeable variables $\left\{Y_{n}^{M}\right\}$ when $M \rightarrow \infty$.

\section{The proof}

In the following proof, since $K$ is always finite and all considered random variables are exchangeable, as above, we will sometimes omit the neuron index $i \in\{1, \ldots, K\}$ in order to simplify notation. Tilde superscripts will refer to objects in the infinite replica limit. Hat superscripts will refer to fragmentation processes.

\section{Step one: fragmentation}

Lemma 9. Let $\hat{X}=\left\{\hat{X}_{n, i}^{M}=X_{n, i}^{M} \mathbb{I}_{\left\{U_{n, i}>\sigma\left(X_{n, i}^{M}\right)\right\}}\right\}$. Then $\operatorname{PAI}(X)$ implies $\operatorname{PAI}(\hat{X})$.

Proof. We have for $u, v \in[0,1]$,

$$
\mathbf{E}\left[u^{\hat{X}_{1}^{M}} v^{\hat{X}_{2}^{M}}\right]=\sum_{k, l \in \mathbb{N}} \mathbf{P}\left(\hat{X}_{1}^{M}=k, \hat{X}_{2}^{M}=l\right) u^{k} v^{l} .
$$

For $k, l>0$, we have

$$
\mathbf{P}\left(\hat{X}_{1}^{M}=k, \hat{X}_{2}^{M}=l\right)=\mathbf{P}\left(X_{1}^{M}=k, X_{2}^{M}=l\right)(1-\sigma(k))(1-\sigma(l)) .
$$

Similarly, we have for $k>0$

$$
\mathbf{P}\left(\hat{X}_{1}^{M}=k, \hat{X}_{2}^{M}=0\right)=\sum_{l \in \mathbb{N}} \mathbf{P}\left(X_{1}^{M}=k, X_{2}^{M}=l\right)(1-\sigma(k)) \sigma(l)
$$


and

$$
\mathbf{P}\left(\hat{X}_{1}^{M}=0, \hat{X}_{2}^{M}=0\right)=\sum_{k, l \in \mathbb{N}} \mathbf{P}\left(X_{1}^{M}=k, X_{2}^{M}=l\right) \sigma(k) \sigma(l)
$$

Since $\operatorname{PAI}(X)$ holds, for all $k, l \in \mathbb{N}, \mathbf{P}\left(X_{1}^{M}=k, X_{2}^{M}=l\right) \rightarrow \mathbf{P}(\tilde{X}=k) \mathbf{P}(\tilde{X}=l)$ when $M \rightarrow \infty$. Since all considered functions are bounded by 1 , we have that for all $k, l \in \mathbb{N}$,

$$
\mathbf{P}\left(\hat{X}_{1}^{M}=k, \hat{X}_{2}^{M}=l\right) \rightarrow \mathbf{P}(\tilde{\hat{X}}=k) \mathbf{P}(\tilde{\hat{X}}=l)
$$

when $M \rightarrow \infty$, where $\tilde{\hat{X}}=\tilde{X} \mathbb{1}_{\{U>\sigma(\tilde{X})\}}$. This shows that

$$
\mathbf{E}\left[u^{\hat{X}_{1}^{M}} v^{\hat{X}_{2}^{M}}\right] \rightarrow \mathbf{E}\left[u^{\tilde{\hat{X}}}\right] \mathbf{E}\left[v^{\tilde{\hat{X}}}\right]
$$

when $M \rightarrow \infty$, which concludes the proof.

\section{Step two: asymptotic behavior of the arrivals processes}

We now show that the number of arrivals $A_{n, i}^{M}$ defined in (8) is asymptotically Poisson as the number of replicas goes to infinity. This is precisely the Poisson Hypothesis introduced in [12].

Lemma 10. Supposing that $\operatorname{PAI}(X)$ holds, we have the following convergence in distribution when $M \rightarrow$ $\infty$

$$
A_{n, i}^{M} \rightarrow \operatorname{Poi}((K-1) \theta)
$$

where $\theta=\mathbf{E}[\sigma(\tilde{X})]$.

Proof. Let $z \in[0,1]$. Then

$$
\begin{aligned}
& \mathbf{E}\left[z^{A_{n, i}^{M}}\right]=\mathbf{E}\left[z^{\sum_{m \neq n} \sum_{j \neq i} \mathbf{I}_{\left\{U_{m, j}<\sigma\left(X_{m, j}^{M}\right)\right\}} \mathbf{I}_{\left\{R_{m, j, i}^{M}=n\right\}}}\right] \\
& =\mathbf{E}\left[\prod_{m \neq n} \prod_{j \neq i} \mathbf{E}\left[z^{\mathbf{I}_{\left\{U_{m, j}<\sigma\left(X_{m, j}^{M}\right)\right\}} \mathbf{I}_{\left\{R_{m, j, i}^{M}=n\right\}}} \mid X_{m, j}^{M}, U\right]\right] \\
& =\mathbf{E}\left[\prod_{m \neq n} \prod_{j \neq i}\left(\left(1-\frac{1}{M-1}\right)+\frac{1}{M-1} z^{\mathbf{I}_{\left\{U_{m, j}<\sigma\left(X_{m, j}^{M}\right)\right\}}}\right)\right] \\
& =\mathbf{E}\left[e^{\sum_{m \neq n} \sum_{j \neq i} \log \left(1-\frac{1}{M-1}\left(1-z^{\mathrm{I}_{\left\{U_{m, j}<\sigma\left(X_{m, j}^{M}\right)\right\}}}\right)\right)}\right] .
\end{aligned}
$$

We now give an upper and lower bound for this expression. Since $\log (1-x) \leq-x$ for $x \leq 1$, we have

$$
\mathbf{E}\left[z^{A_{n, i}^{M}}\right] \leq \mathbf{E}\left[e^{-\frac{1}{M-1} \sum_{m \neq n} \sum_{j \neq i}\left(1-z^{\mathrm{I}_{\left\{U_{m, j}<\sigma\left(X_{m, j}^{M}\right)\right\}}}\right)}\right]
$$

Using the generalized TLLN given in Lemma $7, \frac{1}{M-1} \sum_{m \neq n} \sum_{j \neq i}\left(1-z^{\mathbf{I}_{\left\{U_{m, j}<\sigma\left(X_{m, j}^{M}\right)\right\}}}\right) \rightarrow(K-1)(1-\Phi(z))$ in $L^{2}$ when $M \rightarrow \infty$ with $\Phi(z)=\mathbf{E}\left[z^{\left.\mathbf{I}_{U<\sigma(\tilde{X})}\right]}\right.$, where $U$ is any $U_{m, j}$.

We have $\Phi(z)=z \int_{0}^{1} \mathbf{P}(\sigma(\tilde{X})>t) \mathrm{d} t+\left(1-\int_{0}^{1} \mathbf{P}(\sigma(\tilde{X})>t) \mathrm{d} t\right)=(z-1) \theta+1$. 
Therefore, since $L^{2}$ convergence implies convergence in distribution and thus convergence of the Laplace transforms, $\mathbf{E}\left[e^{-\frac{1}{M-1} \sum_{m \neq n} \sum_{j \neq i}\left(1-z^{I_{\left\{U_{m, j}<\sigma\left(X_{m, j}^{M}\right)\right\}}}\right)}\right] \rightarrow e^{-\theta(1-z)(K-1)}$ when $M \rightarrow \infty$. Thus,

$$
\limsup _{M \rightarrow \infty} \mathbf{E}\left[z^{A_{n, i}^{M}}\right] \leq e^{-\theta(1-z)(K-1)} .
$$

Similarly, since $\log (1-x) \geq-x-\frac{x^{2}}{2}$ for $x \leq 1$, we have

$$
\mathbf{E}\left[z^{A_{n, i}^{M}}\right] \geq \mathbf{E}\left[e^{-\frac{1}{M-1} \sum_{m \neq n} \sum_{j \neq i}\left(1-z^{\mathrm{I}_{\left\{U_{m, j}<\sigma\left(X_{m, j}^{M}\right)\right\}}}\right)-\frac{1}{2(M-1)^{2}} \sum_{m \neq n} \sum_{j \neq i}\left(1-z^{\mathrm{I}_{\left\{U_{m, j}<\sigma\left(X_{m, j}^{M}\right)\right\}}}\right)^{2}}\right] .
$$

Using once again Lemma 7 , as the second term goes to 0 when $M \rightarrow \infty$, by the same reasoning as previously, we get

$$
\liminf _{M \rightarrow \infty} \mathbf{E}\left[z^{A_{n, i}^{M}}\right] \geq e^{-\theta(1-z)(K-1)} .
$$

Combining (20) and (21), the result follows.

Now, we show that the arrivals to different replicas become pairwise asymptotically independent:

Lemma 11. For all $(n, i) \neq(m, j), A_{n, i}^{M}$ and $A_{m, j}^{M}$ are pairwise asymptotically independent.

Proof. We first show the result in the case $n \neq m$ and $i \neq j$. Let $u, v \in[0,1]$. Then

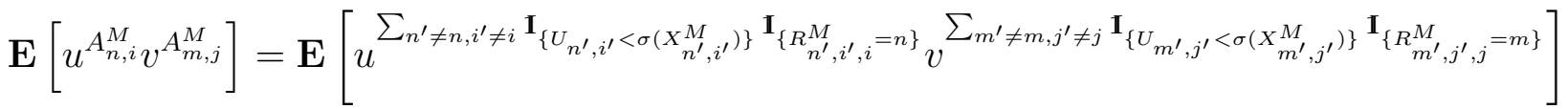

$$
\begin{aligned}
& =\mathbf{E}\left[\prod_{n^{\prime} \neq n, i^{\prime} \neq i} u^{\mathbf{I}_{\left\{U_{n^{\prime}, i^{\prime}}<\sigma\left(X_{n^{\prime}, i^{\prime}}^{M}\right)\right\}} \mathbf{I}_{\left\{R_{n^{\prime}, i^{\prime}, i}^{M}=n\right\}}} \prod_{m^{\prime} \neq m, j^{\prime} \neq j} v^{\left.\mathbf{I}_{\left\{U_{m^{\prime}, j^{\prime}}<\sigma\left(X_{m^{\prime}, j^{\prime}}^{M}\right)\right\}} \mathbf{I}_{\left\{R_{m^{\prime}, j^{\prime}, j}^{M}\right.}=m\right\}}\right] \\
& =\mathbf{E}\left[\mathbf{E}\left[\prod_{n^{\prime} \neq n, i^{\prime} \neq i} u^{\mathbf{I}_{\left\{U_{n^{\prime}, i^{\prime}}<\sigma\left(X_{n^{\prime}, i^{\prime}}^{M}\right)\right\}} \mathbf{I}_{\left\{R_{n^{\prime}, i^{\prime}, i}^{M}=n\right\}}} \prod_{m^{\prime} \neq m, j^{\prime} \neq j} v^{\left.\mathbf{I}_{\left\{U_{m^{\prime}, j^{\prime}}<\sigma\left(X_{m^{\prime}, j^{\prime}}^{M}\right)\right\}} \mathbf{I}_{\left\{R_{m^{\prime}, j^{\prime}, j}^{M}\right.}^{M}=m\right\}} \mid X^{M}, U\right]\right] \\
& =\mathbf{E}\left[\prod_{n^{\prime} \neq n, i^{\prime} \neq i}\left[\left(1-\frac{1}{M-1}\right)+\frac{1}{M-1} u^{\mathbf{I}_{\left\{U_{n^{\prime}, i^{\prime}}<\sigma\left(X_{n^{\prime}, i^{\prime}}^{M}\right)\right\}}}\right]\right. \\
& \cdot \prod_{m^{\prime} \neq m, j^{\prime} \neq j}\left[\left(1-\frac{1}{M-1}\right)+\frac{1}{M-1} v^{\left.\mathbf{I}_{\left\{U_{m^{\prime}, j^{\prime}}<\sigma\left(X_{m^{\prime}, j^{\prime}}^{M}\right)\right\}}\right]}\right] \\
& =\mathbf{E}\left[e^{\sum_{n^{\prime} \neq n, i^{\prime} \neq i} \log \left(1-\frac{1}{M-1}\left(1-u^{\mathrm{I}_{\left\{U_{n^{\prime}, i^{\prime}}<\sigma\left(X_{n^{\prime}, i^{\prime}}^{M}\right)\right\}}}\right)\right)} e^{\sum_{m^{\prime} \neq m, j^{\prime} \neq j} \log \left(1-\frac{1}{M-1}\left(1-v^{\mathrm{I}_{\left\{U_{m^{\prime}, j^{\prime}}<\sigma\left(X_{m^{\prime}, j^{\prime}}\right)\right\}}}\right)\right)}\right] .
\end{aligned}
$$

The fourth equality above comes from the independence between the routing variables $R^{M}$.

Just as in the proof of Lemma 10, we can give upper and lower bounds of the last right-hand side expression:

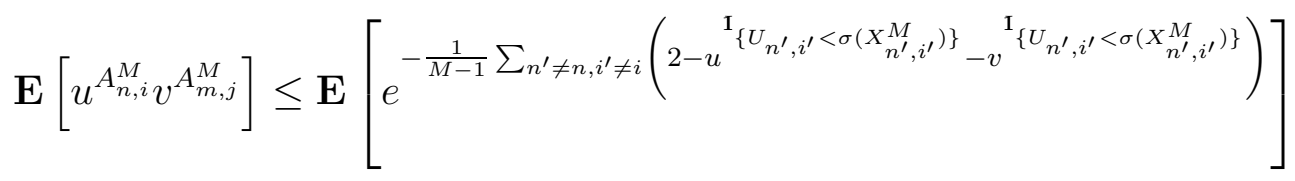

and

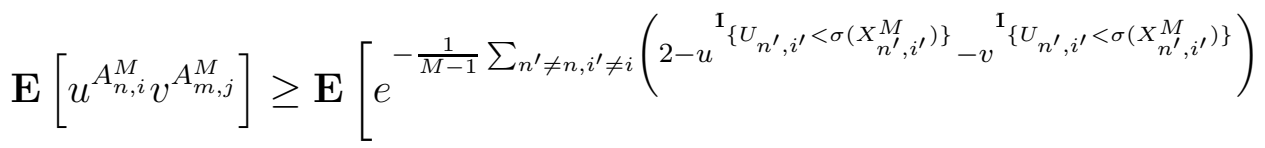

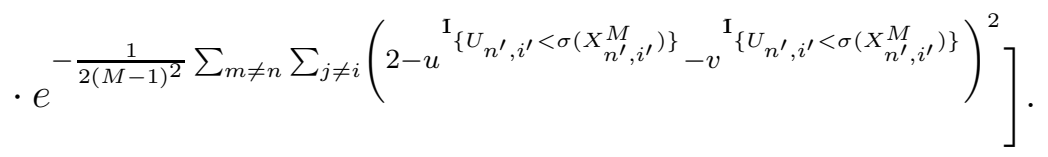


The last right-hand side expression goes to $e^{(1-u+1-v)(K-1) \theta}$ when $M \rightarrow \infty$ in both cases, as previously. The result follows from these two bounds as in the proof of Lemma 10.

The case where $n=m$, i.e., when we consider the arrivals to two different neurons in the same replica, is done in the same way since the routing variables are independent from the neurons chosen. The case where $i=j$, i.e. when we consider the arrivals to the same neuron in two different replicas, is treated in the same way, with the extra step of isolating the terms that are not independent from each other.

\section{Step three: propagation of pairwise asymptotic independence}

Our goal is now to combine the previous results to show that $\operatorname{PAI}(Y)$ holds, assuming $\operatorname{PAI}(X)$. We have that for all $i \in\{1, \ldots, K\}$ and all $n \in\{1, \ldots, M\}, Y_{n, i}^{M}=\hat{X}_{n, i}^{M}+A_{n, i}^{M}$. We call $\tilde{A}$ the limit in distribution of $A_{n, i}^{M}$ (it is Poisson distributed by the previous lemma). It is clear that by exchangeability between replicas, we only require the following lemma:

Lemma 12. For $i, j \in\{1, \ldots, K\}$,

$$
\mathbf{E}\left[u^{Y_{1, i}^{M}}, v^{Y_{2, j}^{M}}\right] \rightarrow \mathbf{E}\left[u^{\tilde{Y}}\right] \mathbf{E}\left[v^{\tilde{Y}}\right]
$$

when $M \rightarrow \infty$, where $\tilde{Y}=\tilde{\hat{X}}+\tilde{A}$.

Proof. Let $u, v \in[0,1]$. Then, given $i, j \in[0, K]$, with $i \neq j$ for simplicity,

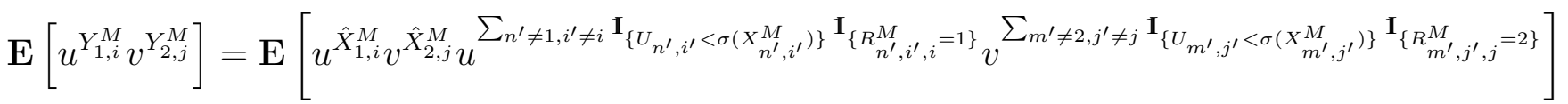

$$
\begin{aligned}
& =\mathbf{E}\left[u^{\hat{X}_{1, i}^{M}} v^{\hat{X}_{2, j}^{M}} \prod_{n^{\prime} \neq 1, i^{\prime} \neq i} u^{\mathbf{I}_{\left\{U_{n^{\prime}, i^{\prime}}<\sigma\left(X_{n^{\prime}, i^{\prime}}^{M}\right)\right\}} \mathbf{I}_{\left\{R_{n^{\prime}, i^{\prime}, i}^{M}=1\right\}}} \prod_{m^{\prime} \neq 2, j^{\prime} \neq j} v^{\left.\mathbf{I}_{\left\{U_{m^{\prime}, j^{\prime}}<\sigma\left(X_{m^{\prime}, j^{\prime}}^{M}\right)\right\}} \mathbf{I}_{\left\{R_{m^{\prime}, j^{\prime}, j}=2\right\}}\right]}\right. \\
& =\mathbf{E}\left[\mathbf{E}\left[u^{\hat{X}_{1, i}^{M} v^{\hat{X}_{2, j}^{M}}} \prod_{n^{\prime} \neq 1, i^{\prime} \neq i} u^{\left.\mathbf{I}_{\left\{U_{n^{\prime}, i^{\prime}}<\sigma\left(X_{n^{\prime}, i^{\prime}}^{M}\right)\right\}} \mathbf{I}_{\left\{R_{n^{\prime}, i^{\prime}, i}^{M}\right.}=1\right\}} \prod_{m^{\prime} \neq 2, j^{\prime} \neq j} v^{\left.\mathbf{I}_{\left\{U_{m^{\prime}, j^{\prime}}<\sigma\left(X_{m^{\prime}, j^{\prime}}^{M}\right)\right\}} \mathbf{I}_{\left\{R_{m^{\prime}, j^{\prime}, j}^{M}\right.}^{M}=2\right\}} \mid X^{M}, U\right]\right]
\end{aligned}
$$

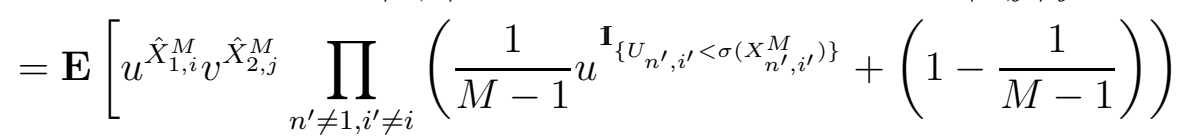

$$
\begin{aligned}
& \left.\prod_{m^{\prime} \neq 2, j^{\prime} \neq j}\left(\frac{1}{M-1} v^{\mathbf{I}_{\left\{U_{m^{\prime}, j^{\prime}}<\sigma\left(X_{m^{\prime}, j^{\prime}}^{M}\right)\right\}}}+\left(1-\frac{1}{M-1}\right)\right)\right] \\
& =\mathbf{E}\left[u^{\hat{X}_{1, i}^{M}}\left(1-\frac{1}{M-1}+\frac{1}{M-1} v^{\mathbf{I}_{\left\{U_{1, i}<\sigma\left(X_{1, i}^{M}\right)\right\}}}\right) v^{\hat{X}_{2, j}^{M}}\left(1-\frac{1}{M-1}+\frac{1}{M-1} u^{\mathbf{I}_{\left\{U_{2, j}<\sigma\left(X_{2, j}^{M}\right)\right\}}}\right)\right. \\
& \left.\cdot e^{\sum_{n^{\prime} \neq 1 ; i^{\prime} \neq i ;\left(n^{\prime}, i^{\prime}\right) \neq(2, j)} \log \left(1-\frac{1}{M-1}\left(1-u^{\left.{ }^{1} U_{n^{\prime}, i^{\prime}}<\sigma\left(X_{n^{\prime}, i^{\prime}}^{M}\right)\right\}}\right)\right)} e^{\sum_{m^{\prime} \neq 2 ; j^{\prime} \neq j ;\left(m^{\prime}, j^{\prime}\right) \neq(1, i)} \log \left(1-\frac{1}{M-1}\left(1-v^{\left.{ }_{\left\{U_{m^{\prime}, j^{\prime}}<\sigma\left(X_{m^{\prime}, j^{\prime}}\right)\right\}}\right)}\right)\right.}\right] \\
& =\mathbf{E}\left[\varphi_{1}^{M}(u, v) \varphi_{2}^{M}(u, v)\right],
\end{aligned}
$$

where $\varphi_{1}^{M}(u, v)=u^{\hat{X}_{1, i}^{M}}\left(1-\frac{1}{M-1}+\frac{1}{M-1} v^{\mathbf{I}_{\left\{U_{1, i}<\sigma\left(X_{1, i}^{M}\right)\right\}}}\right) v^{\hat{X}_{2, j}^{M}}\left(1-\frac{1}{M-1}+\frac{1}{M-1} u^{\mathbf{I}_{\left\{U_{2, j}<\sigma\left(X_{2, j}^{M}\right)\right\}}}\right)$ and

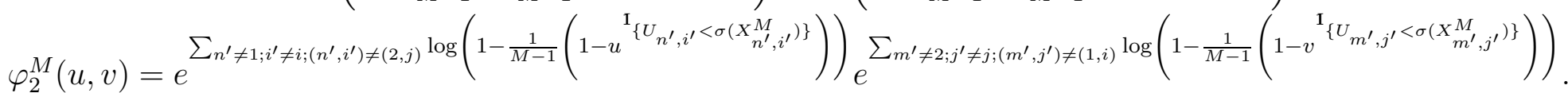

When $M \rightarrow \infty$, by Lemmas 9 and 11, $\varphi_{1}^{M}(u, v)$ and $\varphi_{2}^{M}(u, v)$ are pairwise asymptotically independent. Since in $\varphi_{2}^{M}(u, v)$, the contribution of the missing terms in the sum is negligible, when $M \rightarrow \infty$, we have

$$
\mathbf{E}\left[\varphi_{1}^{M}(u, v) \varphi_{2}^{M}(u, v)\right] \rightarrow \mathbf{E}\left[u^{\tilde{\hat{X}}}\right] \mathbf{E}\left[v^{\tilde{\hat{X}}}\right] \mathbf{E}\left[u^{\tilde{\hat{A}}}\right] \mathbf{E}\left[v^{\tilde{\hat{A}}}\right]
$$

This shows that (22) holds. 
Thus, $\operatorname{PAI}(X)$ implies $\operatorname{PAI}(Y)$, which concludes the proof of the theorem. Note that Lemma 12 also shows that $\tilde{\hat{X}}$ and $\tilde{A}$ are independent.

\section{The symmetric fragmentation-interaction-aggregation process}

Our goal is to show that propagation of chaos and the Poisson hypothesis hold in the more general setting of symmetric FIAPs under mild hypotheses on the dynamics of the system. The symmetrical evolution equations read

$$
Y_{i}=g_{1}\left(X_{i}\right) \mathbb{I}_{\left\{U_{i}<\sigma\left(X_{i}\right)\right\}}+g_{2}\left(X_{i}\right) \mathbb{I}_{\left\{U_{i}>\sigma\left(X_{i}\right)\right\}}+A_{i}
$$

where

$$
A_{i}=\sum_{j \neq i} h\left(X_{j}\right) \mathbb{1}_{\left\{U_{j}<\sigma\left(X_{j}\right)\right\}}
$$

and $g_{1}, g_{2}, h: \mathbb{N} \rightarrow \mathbb{N}$ are functions such that $h$ is bounded.

We now introduce the corresponding replica dynamics. Let $\left\{X_{n, i}^{M}\right\}$ be the integer-valued state variables at step 0 , where $n \in\{1, \ldots, M\}$ and $i \in\{1, \ldots, K\}$. Let $\left\{Y_{n, i}^{M}\right\}$ be the integer-valued state variables at time one. Let $\left\{U_{n, i}\right\}$ be i.i.d. random variables independent from $\left\{X_{n, i}^{M}\right\}$ uniformly distributed in $[0,1]$. We introduce again the i.i.d. routing variables $R_{m, j, i}^{M}$, independent from $\left\{U_{n, i}\right\}$ and $\left\{X_{n, i}^{M}\right\}$ and uniformly distributed in $\{1, \ldots, M\} \backslash\{m\}$. The $M$-replica equations read:

$$
Y_{n, i}^{M}=g_{1}\left(X_{n, i}^{M}\right) \mathbb{I}_{\left\{U_{n, i}<\sigma\left(X_{n, i}^{M}\right)\right\}}+g_{2}\left(X_{n, i}^{M}\right) \mathbb{I}_{\left\{U_{n, i}>\sigma\left(X_{n, i}^{M}\right)\right\}}+A_{n, i}^{M},
$$

where

$$
A_{n, i}^{M}=\sum_{m \neq n} \sum_{j \neq i} h\left(X_{m, j}^{M}\right) \mathbb{I}_{\left\{U_{m, j}<\sigma\left(X_{m, j}^{M}\right)\right\}} \mathbb{I}_{\left\{R_{m, j, i}^{M}=n\right\}}
$$

is the number of arrivals in node $i$ of replica $n$.

We also recall the definition of a compound Poisson distribution:

Definition 13. The random variable $X$ is said to follow a compound Poisson distribution if there exist a Poisson $(\lambda)$ random variable $N$ and i.i.d. random variables $\left(X_{i}\right)_{i \in \mathbb{N} \star}$ independent from $N$ such that

$$
X=\sum_{i=1}^{N} X_{i}
$$

The generating function of $X$, denoted $\varphi_{X}$, is given by

$$
\varphi_{X}(t)=e^{\lambda(\varphi(t)-1)},
$$

where $\varphi(t)$ is the generating function of $X_{1}$.

We have the following theorem:

Theorem 14. For all symmetric RMF FIAP dynamics, $\operatorname{PAI}(X)$ implies $\operatorname{PAI}(Y)$. Moreover, the arrivals to a given node are asymptotically compound Poisson distributed.

We will require the following lemmas. The following result replaces Lemma 9:

Lemma 15. Let $\hat{X}^{1}=\left\{\hat{X}_{n, i}^{1, M}=g_{1}\left(X_{n, i}^{M}\right) \mathbb{I}_{\left\{U_{n, i}<\sigma\left(X_{n, i}^{M}\right)\right\}}\right\}$. Let $\hat{X}^{2}=\left\{\hat{X}_{n, i}^{2, M}=g_{2}\left(X_{n, i}^{M}\right) \mathbb{I}_{\left\{U_{n, i}>\sigma\left(X_{n, i}^{M}\right)\right\}}\right\}$. Then $\operatorname{PAI}(X)$ implies $\operatorname{PAI}\left(\hat{X}^{1}\right), \operatorname{PAI}\left(\hat{X}^{2}\right)$ and $\operatorname{PAI}(\hat{X})$, where $\hat{X}=\hat{X}^{1}+\hat{X}^{2}$. 
Proof. We proceed exactly as in Lemma 9. We write here only the proof for $\hat{X}^{2}$, the others being identical except for the numerical expressions involved. We have for $u, v \in[0,1]$,

$$
\mathbf{E}\left[u^{\hat{X}_{1}^{2, M}} v^{\hat{X}_{2}^{2, M}}\right]=\sum_{k, l \in \mathbb{N}} \mathbf{P}\left(\hat{X}_{1}^{2, M}=k, \hat{X}_{2}^{2, M}=l\right) u^{k} v^{l} .
$$

For $k, l>0$, we have

$$
\mathbf{P}\left(\hat{X}_{1}^{2, M}=k, \hat{X}_{2}^{2, M}=l\right)=\sum_{p, q \in \mathbb{N}} \mathbf{P}\left(g_{2}\left(X_{1}^{M}\right)=k, g_{2}\left(X_{2}^{M}\right)=l, X_{1}^{M}=p, X_{2}^{M}=q\right)(1-\sigma(p))(1-\sigma(q)) .
$$

Since $\operatorname{PAI}(X)$ holds, $\mathbf{P}\left(g_{2}(p)=k, g_{2}(q)=l, X_{1}^{M}=p, X_{2}^{M}=q\right) \rightarrow \mathbf{P}\left(\tilde{X}=p, g_{2}(p)=k\right) \mathbf{P}\left(\tilde{X}=q, g_{2}(q)=l\right)$ when $M \rightarrow \infty$. Hence, $\mathbf{E}\left[u^{\hat{X}_{1}^{2, M}} v^{\hat{X}_{2}^{2, M}}\right] \rightarrow \mathbf{E}\left[u^{g_{2}(\tilde{X}) \mathbf{I}_{\{U<\sigma(\tilde{X})\}}}\right] \mathbf{E}\left[v^{g_{2}(\tilde{X}) \mathbf{I}_{\{U<\sigma(\tilde{X})\}}}\right]$ when $M \rightarrow \infty$. The cases where $k$ and/or $l$ are equal to 0 are handled in the same way. This proves the result.

We now prove the following result, which replaces Lemma 10:

Lemma 16. Supposing that $\operatorname{PAI}(X)$ holds, we have the following convergence in distribution when $M \rightarrow$ $\infty$

$$
A_{n, i}^{M} \rightarrow \tilde{A}
$$

where $\tilde{A}$ follows a compound Poisson distribution.

Proof. We still have, just like in the proof of Lemma 10, that for $z \in[0,1], i \in\{1, \ldots, K\}, n \in\{1, \ldots, M\}$,

$$
\mathbf{E}\left[z^{A_{n, i}^{M}}\right]=\mathbf{E}\left[e^{\sum_{m \neq n} \sum_{j \neq i} \log \left(1-\frac{1}{M-1}\left(1-z^{h\left(X_{m, j}^{M}\right) \mathbf{I}_{\left\{U_{m, j}<\sigma\left(X_{m, j}^{M}\right)\right\}}}\right)\right)}\right]
$$

Using the same arguments as before, we have when $M \rightarrow \infty$

$$
\mathbf{E}\left[z^{A_{n, i}^{M}}\right] \rightarrow e^{(K-1)(\Phi(z)-1)}
$$

where $\Phi(z)=\mathbf{E}\left[z^{h(\tilde{X}) \mathbf{I}_{\{U<\sigma(\tilde{X})\}}}\right]$, which is precisely of the form (28), that is, a generating function of a random variable with a compound Poisson distribution.

We now combine these results to prove Theorem 14 .

Proof. We follow the outline of the previous section. Lemmas 5, 6 and 7 still apply as previously. Lemma 15 replaces Lemma 9. Lemma 16 replaces Lemma 10. Lemmas 11 and 12 still hold, with only differences in the limiting expressions.

\section{The general fragmentation-interaction-aggregation process}

The previously introduced exchangeable dynamics allow for simpler computations at the expense of realistic modeling. For example, neuron populations are not homogeneous and are not fully connected. In order to account for such a geometry, we now generalize the previous result to the case where the functions governing the information received by a node when another node activates depend on the nodes involved. Specifically, recall the class $\mathcal{C}$ of discrete FIAP defined in Section 1.

For any process in $\mathcal{C}$, we can define a replica mean field model as in the previous sections: we consider a collection of $M$ identically distributed replicas of a set of $K$ nodes, which could be neurons, particles, queues or other objects, depending on context. As previously, let $\left\{X_{n, i}^{M}\right\}$ be the integer-valued state variables at 
step 0 , where $n \in\{1, \ldots, M\}$ and $i \in\{1, \ldots, K\}$. Let $\left\{Y_{n, i}^{M}\right\}$ be the integer-valued state variables at time one. Let $\left\{U_{n, i}\right\}$ be uniformly distributed on $[0,1]$ i.i.d. random variables independent from $\left\{X_{n, i}^{M}\right\}$. Let $\left\{R_{m, j, i}^{M}\right\}$ be i.i.d. routing random variables independent from $\left\{X_{n, i}^{M}\right\}$ and $\left\{U_{n, i}\right\}$, uniformly distributed on $\{1, \ldots, M\} \backslash\{m\}$ for all $i, j \in\{1, \ldots, K\}$ and $m \in\{1, \ldots, M\}$. Recall that the $M$-RMF equations read

$$
Y_{n, i}^{M}=g_{1, i}\left(X_{n, i}^{M}\right) \mathbb{I}_{\left\{U_{n, i}<\sigma_{i}\left(X_{n, i}^{M}\right)\right\}}+g_{2, i}\left(X_{n, i}^{M}\right) \mathbb{1}_{\left\{U_{n, i}>\sigma_{i}\left(X_{n, i}^{M}\right)\right\}}+A_{n, i}^{M},
$$

where

$$
A_{n, i}^{M}=\sum_{m \neq n} \sum_{j \neq i} h_{i, j}\left(X_{m, j}^{M}\right) \mathbb{I}_{\left\{U_{m, j}<\sigma_{i}\left(X_{m, j}^{M}\right)\right\}} \mathbb{I}_{\left\{R_{m, j, i}^{M}=n\right\}}
$$

is the number of arrivals in node $i$ of replica $n$. We now show that the result from the previous section carries over to this more general setting with only minor modifications.

First, we must slightly modify the definition of pairwise asymptotic independence in order to take into account the dependence on the node of the limiting distribution. As a simplification, we keep the same notations for this modified definition.

Definition 17. Given $M \in \mathbb{N}$, given an array of integer-valued random variables $Z=\left\{Z_{n, i}^{M}\right\}_{1 \leq n \leq M, 1 \leq i \leq K}$ such that for all fixed $M$, the random variables $Z_{n, i}^{M}$ are exchangeable in $n$, we say that the variables $Z_{n, i}^{M}$ are pairwise asymptotically independent, which we will denote $\operatorname{PAI}(Z)$, if there exist integer-valued random variables $\left(\tilde{Z}_{i}\right)_{i \in\{1, \ldots, K\}}$ such that $\forall(n, i) \neq(m, j), \forall u, v \in[0,1]$,

$$
\lim _{M \rightarrow \infty} \mathbf{E}\left[u^{Z_{n, i}^{M}} v^{Z_{m, j}^{M}}\right]=\mathbf{E}\left[u^{\tilde{Z}_{i}}\right] \mathbf{E}\left[v^{\tilde{Z}_{j}}\right]
$$

For clarity of exposition, we also recall here the definition of the triangular law of large numbers, even though it is left unchanged:

Definition 18. Given $M \in \mathbb{N}$, given an array of integer-valued random variables $Z=\left\{Z_{n}^{M}\right\}_{n \in\{1, \ldots, M\}}$ such that for all fixed $M$, the random variables $Z_{n}^{M}$ are exchangeable in $n$, we say that $Z$ verifies the triangular law of large numbers $\operatorname{TLLN}(Z)$ if there exist an integer-valued random variable $\tilde{Z}$ such that for all functions $f: \mathbb{N} \rightarrow \mathbb{R}$ with compact support, we have the following limit in $L^{2}$ :

$$
\lim _{M \rightarrow \infty} \frac{1}{M} \sum_{n=1}^{M} f\left(Z_{n}^{M}\right)=\mathbf{E}[f(\tilde{Z})] .
$$

Then, we obtain the same result:

Theorem 19. Using previously defined notations, $\operatorname{PAI}(X)$ implies $\operatorname{PAI}(Y)$. Moreover, the arrivals to a given node are asymptotically compound Poisson distributed and are independent of the states of the nodes.

We once again require the following lemmas for the proof.

We replace Lemma 7 with the following similar result, taking into account the fact that the limiting distribution now depends on the node:

Lemma 20. Let $M \in \mathbb{N}$, let $Z=\left\{Z_{n, i}^{M}\right\}_{n \in\{1, \ldots, M\}, i \in\{1, \ldots, K\}}$ be an array of integer valued random variables verifying $\operatorname{PAI}(Z)$. Then for all bounded functions $f: \mathbb{N} \times[0,1] \rightarrow \mathbb{R}$ with compact support, for all i.i.d. sequences of random variables $U=\left\{U_{n, i}\right\}_{n \in\{1, \ldots, M\}, i \in\{1, \ldots, K\}}$ independent from $Z$, there exists $U$ independent from $\left(\tilde{Z}_{i}\right)_{i \in\{1, \ldots, K\}}$ and $Z$ such that for all $i \in\{1, \ldots, K\}$, we have the following limit in $L^{2}$ :

$$
\lim _{M \rightarrow \infty} \frac{1}{M} \sum_{n=1}^{M} f\left(Z_{n, i}^{M}, U_{n, i}\right)=\mathbf{E}\left[f\left(\tilde{Z}_{i}, U\right)\right] .
$$


The proof is exactly the same as for Lemma 6 .

We must replace Lemma 10 with the following result:

Lemma 21. Supposing that $\operatorname{PAI}(X)$ holds, we have the following convergence in distribution when $M \rightarrow$ $\infty$ :

$$
A_{n, i}^{M} \rightarrow \tilde{A}_{i}
$$

where $\tilde{A}_{i}$ follows a compound Poisson distribution.

Proof. We have for $z \in[0,1], i \in\{1, \ldots, K\}, n \in\{1, \ldots, M\}$, that

$$
\mathbf{E}\left[z^{A_{n, i}^{M}}\right]=\mathbf{E}\left[e^{\sum_{m \neq n} \sum_{j \neq i} \log \left(1-\frac{1}{M-1}\left(1-z^{h_{i, j}\left(X_{m, j}^{M}\right) \mathbf{I}_{\left\{U_{m, j}<\sigma_{i}\left(X_{m, j}^{M}\right)\right\}}}\right)\right.}\right] .
$$

Using the same arguments as before, we have when $M \rightarrow \infty$

$$
\mathbf{E}\left[z^{A_{n, i}^{M}}\right] \rightarrow e^{\Phi_{i}(z)}
$$

where $\Phi_{i}(z)=-\sum_{j \neq i} \mathbf{E}\left[1-z^{h_{i, j}\left(\tilde{X}_{i}\right) \mathbf{I}_{\left\{U<\sigma_{i}\left(\tilde{X}_{i}\right)\right\}}}\right]$. Therefore,

$$
\mathbf{E}\left[z^{A_{n, i}^{M}}\right] \rightarrow e^{-\sum_{j \neq i}\left(1-\mathbf{E}\left[z^{h_{i, j}\left(\tilde{X}_{i}\right) \mathbf{I}_{\left\{U<\sigma_{i}\left(\tilde{X}_{i}\right)\right\}}}\right]\right)}
$$

The expression is of the form (28), which proves Lemma 21.

We now prove Theorem 19 .

Proof. We use the same reasoning as previously.

Lemma 6 still holds (the replicas are still exchangeable, only the nodes are not). Lemma 20 replaces Lemma 7. Since the functions $g_{1, i}$ and $g_{2, i}$ only depend on the node and not on the replica index, an equivalent result to Lemma 15 still holds. Lemma 10 is replaced by Lemma 21. For asymptotic independence, we have, using the same arguments as in the proof of Lemma 11, that for $u, v \in[0,1]$, for $n \neq m$ and $i \neq j$,

$$
\mathbf{E}\left[u^{A_{n, i}^{M}} v^{A_{m, j}^{M}}\right] \rightarrow e^{-\sum_{i^{\prime} \neq i}\left(1-\mathbf{E}\left[u^{h_{i, i^{\prime}}\left(\tilde{X}_{i}\right) \mathbf{I}_{\left\{U<\sigma_{i}\left(\tilde{X}_{i}\right)\right\}}}\right]\right)-\sum_{j^{\prime} \neq j}\left(1-\mathbf{E}\left[v^{h_{j, j^{\prime}}\left(\tilde{X}_{j}\right) \mathbf{I}_{\left\{U<\sigma_{i}\left(\tilde{X}_{j}\right)\right\}}}\right]\right)},
$$

when $M \rightarrow \infty$. The other cases $(n=m$ and $i=j)$ are also valid. Lemma 12 also still holds, with only minor differences in the limit expressions.

Note that once again, this proves that the limit processes $\tilde{\hat{X}}_{i}$ and $\tilde{A}_{j}$ are independent for all $i, j \in$ $\{1, \ldots, K\}$.

As an application, let us apply this result to the model from Section 2 with the addition of nonexchangeable interactions. Namely, we consider $h_{i, j}\left(X_{m, j}^{M}\right)=\mu_{i, j}$ with $\mu_{i, j} \in \mathbb{N}$ (potentially zero). In this case, Theorem 19 proves the propagation of chaos in this system, and the limit distributions of arrivals at the different nodes are characterized by, for $i \in\{1, \ldots, K\}$ and $z \in[0,1]$,

$$
\mathbf{E}\left[z^{\tilde{A}_{i}}\right]=e^{\theta_{i} \sum_{j \neq i}\left(z^{\mu_{i, j}}-1\right)}=\prod_{j \neq i} e^{\theta_{i}\left(z^{\mu_{i, j}}-1\right)}
$$

where $\theta_{i}=\mathbf{E}\left[\sigma_{i}\left(\tilde{X}_{i}\right)\right]$. Note that as expected, when all $\mu_{i, j}$ are equal to one, we obtain the result from Section 2. 


\section{Extensions}

There are several ways of extending the FIAP framework while preserving the basic properties proved in the present paper (propagation of chaos and Poisson hypothesis). We decided not to include them in the general framework in order to keep notation and exposition light. A few natural extensions of this type are nevertheless discussed below.

- Random Interactions: the functions $h_{i, j}(k)$ can be replaced by randomized functions of the type $h_{i, j}\left(k, V_{i, j}\right)$ where the random variables $\left\{V_{i, j}\right\}_{1 \leq i, j \leq K}$ are uniform in $[0,1]$ and i.i.d.. This allows one to represent, e.g., the queuing theory scenario where a customer leaving a queue is randomly routed to an other queue of the network according to some stochastic routing matrix $\left\{p_{j, i}\right\}_{1 \leq i, j \leq K}$, namely a customer leaving queue $j$ is routed to queue $j$ with probability $p_{j, i}$. If the random variables $\left\{V_{i, j}\right\}$ are independent of $\left\{X_{i}\right\}_{i}$, then the main results still hold.

- Time inhomogeneous dynamics: The general setting of the paper implicitly suggests to use the same (activation, fragmentation, and interaction) functions at all time steps for a given node. There is no difficulty extending the results to the time inhomogeneous case where these functions depend on the time step. In the neural network case, this for instance happens in certain learning dynamics where the synaptic weights evolve over time.

- Exogenous input: to the endogenous arrivals $A_{i}$ to node $i$ given in Equation (1), we add exogenous arrivals $B_{i}$. If the variables $\left\{B_{i}\right\}$ are independent, Poisson, and independent of the state variables $\left\{X_{i}\right\}_{i}$ then the same results still hold. Note that one can also define an exogenous output for node $i$ through the relation

$$
D_{i}=h_{o, i}\left(X_{i}\right) \mathbb{I}_{\left\{U_{i}<\sigma_{i}\left(X_{i}\right)\right\}},
$$

where $h_{o, i}$ is a given output function $\mathbb{N} \rightarrow \mathbb{N}$.

- Vector State - Example: this extension is first described through a simple neural network example. We partition the set of neurons of a discrete Galves-Löcherbach network in pairs (this assumes that $K$ is even). Each pair of the partition is a node of the network. If $(i, j)$ is one of these nodes, it has a two-dimensional vector state $\left(X_{i}, X_{j}\right)$ (rather than a one dimensional state in the initial model). We let this pair (as well as each other pair in the partition) evolve as a two-node Galves-Löcherbach network with some vectorial exogenous input. Namely, conditionally on $\left(X_{i}, X_{j}\right)=(k, l)$, neurons $i$ and $j$ spike independently with probability $\sigma_{i}(k)$ and $\sigma_{j}(l)$ respectively. If none of them spikes, the state $\left(Z_{i}, Z_{j}\right)$ after its endogenous evolution is still $\left(X_{i}, X_{j}\right)$. If only $i$ (resp. $j$ ) spikes and the other neuron of the pair does not spike, then $\left(Z_{i}, Z_{j}\right)$ is equal $\left(0, X_{j}\right)$ (resp. $\left(X_{i}, 0\right)$ ). If both spike, then $\left(Z_{i}, Z_{j}\right)=(1,1)$. Therefore, if $\left(B_{i}, B_{j}\right)$ denotes the vector exogenous input, the state of this pair at time one is $\left(Z_{i}+B_{i}, Z_{j}+B_{j}\right)$, by combining the endogenous evolution and the exogenous arrivals. Define now the exogenous output of type $k \notin\{i, j\}$ of node $(i, j)$ by

$$
D_{k}(i, j)=\mathbb{I}_{\left\{U_{i}<\sigma_{i}\left(X_{i}\right)\right\}} r_{k, i}+\mathbb{I}_{\left\{U_{j}<\sigma_{j}\left(X_{j}\right)\right\}} r_{k, i},
$$

with $r_{k, i}$ equal to 1 of there is a directed edge from $i$ to $k$ and 0 otherwise. The extension of interest here is that where we take

$$
B_{j}=\sum_{k \notin\{i, j\}} D_{j}(k, l) \mathbb{I}_{\{k<l\}},
$$

with $l$ the node paired with $k$. Note that when node $i$ spikes, the effect on pair $(k, l)$ is as follows: no effect if $r_{k, i}=r_{l, i}=0$; one arrival in $k$ and none in $l$ (resp. one in $l$ and none in $k$ ) if $r_{k, i}=1$ and $r_{l, i}=0$ (resp. $r_{l, i}=1$ and $r_{k, i}=0$ ); a simultaneous arrival in both $l$ and $k$ otherwise. This defines a network which does not belong to the FIAP class. The $M$-RMF model features $M$ replicas of this network with $K / 2$ (vector state) nodes each. In this $M$-RMF model, the exogenous output 
of node/pair $(i, j)$ in replica $m$ is randomly sent to a replica chosen at random. More precisely, for all exogenous output type $k$ paired with $l$,

$D_{k}^{m}(i, j)=\mathbb{I}_{\left\{U_{i}^{m}<\sigma_{i}\left(X_{i}^{m}\right)\right\}} r_{k, i}+\mathbb{I}_{\left\{U_{j}^{m}<\sigma_{j}\left(X_{j}^{m}\right)\right\}} r_{k, i}, \quad\left(\operatorname{resp} . D_{l}^{m}(i, j)=\mathbb{I}_{\left\{U_{i}^{m}<\sigma_{i}\left(X_{i}^{m}\right)\right\}} r_{l, i}+\mathbb{I}_{\left\{U_{j}^{m}<\sigma_{j}\left(X_{j}^{m}\right)\right\}} r_{l, i}\right)$

units are sent to $k$ (resp. $l$ ) of another replica selected uniformly at random where their aggregated to the coordinates of the state variable of this pair. It can be shown that when $M$ tends to infinity,

1. the random state vectors $\left(X_{i}^{m}, X_{j}^{m}\right)$ and $\left(X_{i^{\prime}}^{m}, X_{j^{\prime}}^{m}\right)$, where $(i, j)$ and $\left(i^{\prime}, j^{\prime}\right)$ are two different pairs, are asymptotically independent (although the two coordinates of each vector are in general dependent);

2. the exogenous arrivals to any coordinate of a pair in a typical replica tends to an independent compound Poisson variable.

- Vector State - General Case: Consider a FIAP $F$ with $K$ nodes. Let $S_{1}, S_{2}, \ldots, S_{l}$ be a partition of $[1, \ldots, K]$. Let $K_{p}, 1 \leq p \leq l$ denote the cardinality of set $S_{p}$, and let $F_{p}$ be the restriction of $F$ to the coordinates of $S_{p}$. Let $\mathfrak{F}_{p}$ be the FIAP combining the endogenous dynamics of $F_{p}$ and exogenous input $\left(\mathfrak{B}_{p, i}, i \in S_{p}\right)$. Let $\mathfrak{X}_{p, i}$ denote the state variables in $\mathfrak{F}_{p}$. For all $k \notin S_{p}$, define the exogenous output of type $k$ of $\mathfrak{F}_{p}$ as

$$
\mathfrak{D}_{p}(k)=\sum_{i \in S_{p}} \mathbb{I}_{\left\{U_{p, i}<\sigma_{i}\left(\mathfrak{X}_{p, i}\right)\right\}} h_{k, i}\left(\mathfrak{X}_{p, i}\right)
$$

If we take

$$
\mathfrak{B}_{p, i}=\sum_{q \neq p} \sum_{k \in S_{q}} \mathfrak{D}_{q}(k), i \in S_{p}
$$

we get another (more complex) representation of the dynamics of $F$ based on the point processes describing the interactions between the sets of the partition. The $M$-RMF model associated with this partition features $M$ replicas of this network with $q$ (vector state) nodes each. In this $M$-RMF model, the exogenous output of node $i \in S_{p}$ of replica $m$ is randomly sent to replicas chosen at random. More precisely, for all $q \neq p$, the vector $\left(\mathfrak{D}_{p}^{m}(k), k \in S_{q}\right)$, with $\mathfrak{D}_{p}^{m}(k)$ defined as in (43), is sent to one replica chosen at random, and this is done independently for all $q \neq p$. This in turn defines new exogenous input point processes $\mathfrak{B}_{p, i}^{m}$ as in (44). Let $\left(\mathfrak{X}_{p, i}^{m}, i \in S_{p}, p=1, \ldots, l, m=1, \ldots, M\right)$ denote the state variables in this $M$-RMF model. It can be shown that, when $M$ tends to infinity,

1. for all $p \neq q$, the random state vectors $\left(\mathfrak{X}_{p, i}^{m}, i \in S_{p}\right)$ and $\left(\mathfrak{X}_{q, j}^{m}, j \in S_{q}\right)$, are asymptotically independent (although the coordinates of each vector are in general dependent);

2. for all $p$, and for all $m$, the exogenous arrivals $\left(\mathfrak{B}_{p, i}^{m}, i \in S_{p}\right)$ to set $S_{p}$ tend to an independent multivariate compound Poisson variable with multivariate generating function

$$
\exp \left(\sum_{q \neq p} \sum_{n_{i} \in \mathbb{N}, i \in S_{q}} \sum_{s \subset S_{q}} \pi_{q, s,\left(n_{i}\right)}\left(1-\prod_{i \in s} \prod_{k \in S_{q}} z_{k}^{h_{k, i}\left(n_{i}\right)}\right)\right) .
$$

In this last equation,

$$
\pi_{q, s,\left(n_{i}\right)}=\mathbf{P}\left[\tilde{\mathfrak{X}}_{q, i}=n_{i}, i \in S_{q}\right] \prod_{j \in s} \sigma_{j}\left(n_{j}\right) \prod_{j^{\prime} \in S_{q} \backslash s}\left(1-\sigma_{j^{\prime}}\left(n_{j^{\prime}}\right)\right)
$$

where $\left(\tilde{\mathfrak{X}}_{q, i}\right)$ denotes random variables with the limiting joint distribution assumed in the vector generalization of PAI. 


\section{Conclusion}

A new class of discrete time dynamics involving point process based interactions between interconnected nodes was introduced. The Poisson Hypothesis was proved for the RMF version of such dynamics. The proof is based on the property of pairwise asymptotic independence between replicas and is by induction over time. The key point is that randomized routing decisions on exchangeable events which are asymptotically independent lead to Poisson point processes. The main open questions in relation with this line of thoughts are the extension of the results to time stationary regimes and the extension to continuous time versions of this type of dynamics. 


\section{References}

[1] Frédéric Amblard and Guillaume Deffuant. "The role of network topology on extremism propagation with the relative agreement opinion dynamics". In: Physica A: Statistical Mechanics and its Applications 343 (Nov. 2004), 725-738. ISSN: 0378-4371. DOI: 10.1016/j . physa. 2004.06 .102. URL: http://dx.doi.org/10.1016/j.physa.2004.06.102.

[2] François Baccelli, David R. Mcdonald, and Julien Reynier. A Mean-Field Model for Multiple TCP Connections through a Buffer Implementing RED. Research Report RR-4449. INRIA, 2002. URL: https://hal.inria.fr/inria-00072139.

[3] François Baccelli and Thibaud Taillefumier. "Replica-Mean-Field Limits for Intensity-Based Neural Networks". In: SIAM Journal on Applied Dynamical Systems 18.4 (Jan. 2019), 1756-1797. ISSN: 1536-0040. DOI: 10.1137/19m1243877. URL: http://dx.doi.org/10.1137/19m1243877.

[4] Michel Benaim and Jean-Yves Le Boudec. "A Class Of Mean Field Interaction Models for Computer and Communication Systems". In: vol. 65. May 2008, pp. 589-590. ISBN: 978-963-9799-18-9. DOI: 10.1109/WIOPT. 2008.4586140.

[5] Jean-Yves Le Boudec, David McDonald, and Jochen Mundinger. "A Generic Mean Field Convergence Result for Systems of Interacting Objects". In: Proceedings of the Fourth International Conference on Quantitative Evaluation of Systems. QEST '07. USA: IEEE Computer Society, 2007, 3-18. ISBN: 076952883X. DOI: 10.1109/QEST.2007.3. URL: https://doi.org/10.1109/QEST . 2007.3.

[6] Tommaso Castellani and Andrea Cavagna. "Spin-glass theory for pedestrians". In: Journal of Statistical Mechanics: Theory and Experiment 2005.05 (May 2005), P05012. ISSN: 1742-5468. DOI: 10.1088/ 1742-5468/2005/05/p05012. URL: http://dx.doi.org/10.1088/1742-5468/2005/05/P05012.

[7] Bruno Cessac. "A discrete time neural network model with spiking neurons". In: Journal of Mathematical Biology 56.3 (Sept. 2007), 311-345. ISSN: 1432-1416. DOI: 10.1007/s00285-007-0117-3. URL: http://dx.doi .org/10.1007/s00285-007-0117-3.

[8] Marie Doumic Jauffret and Pierre Gabriel. "Eigenelements of a general aggregation-fragmentation model". In: Mathematical Models and Methods in Applied Sciences 20.05 (May 2010), 757-783. ISSN: 1793-6314. DOI: 10 . 1142 / s021820251000443x. URL: http : / / dx . doi . org / 10 . 1142 / S021820251000443X.

[9] Antonio Galves and Eva Löcherbach. "Infinite Systems of Interacting Chains with Memory of Variable Length-A Stochastic Model for Biological Neural Nets". In: Journal of Statistical Physics 151.5 (Mar. 2013), 896-921. ISSN: 1572-9613. DOI: 10.1007/s10955-013-0733-9. URL: http : //dx.doi.org/10.1007/s10955-013-0733-9.

[10] Nicolas Gast, Diego Latella, and Mieke Massink. "A refined mean field approximation of synchronous discrete-time population models". In: Performance Evaluation 126 (Oct. 2018), 1-21. ISSN: 0166-5316. DOI: 10.1016/j.peva.2018.05.002. URL: http://dx.doi.org/10.1016/j.peva.2018.05.002.

[11] Ankit Gupta, Corentin Briat, and Mustafa Khammash. "A Scalable Computational Framework for Establishing Long-Term Behavior of Stochastic Reaction Networks". In: PLoS Computational Biology 10.6 (June 2014). Ed. by RuthEditor Nussinov, e1003669. ISSN: 1553-7358. DOI: 10.1371/journal. pcbi.1003669. URL: http://dx.doi.org/10.1371/journal.pcbi. 1003669.

[12] Leonard Kleinrock. Communication nets: Stochastic message flow and delay. Courier Corporation, 2007.

[13] Leonard Kleinrock. Queueing Systems. Vol. II: Computer Applications. Wiley Interscience, 1977.

[14] Angelo Masi, Antonio Galves, Eva Löcherbach, and Errico Presutti. "Hydrodynamic Limit for Interacting Neurons". In: Journal of Statistical Physics 158 (2015), pp. 866-902. 
[15] Romualdo Pastor-Satorras, Claudio Castellano, Piet Van Mieghem, and Alessandro Vespignani. "Epidemic processes in complex networks". In: Reviews of Modern Physics 87.3 (Aug. 2015), 925-979. ISSN: 1539-0756. DOI: 10 . $1103 /$ revmodphys . 87 .925. URL: http : / / dx . doi . org/10 . $1103 /$ RevModPhys .87.925.

[16] Youngsoo Seol. "Limit theorems for discrete Hawkes processes". In: Statistics \& Probability Letters 99 (2015), pp. 223-229.

[17] Oren Shriki, Jeff Alstott, Frederick Carver, Tom Holroyd, Richard N.A. Henson, Marie L. Smith, Richard Coppola, Edward Bullmore, and Dietmar Plenz. "Neuronal Avalanches in the Resting MEG of the Human Brain". In: Journal of Neuroscience 33.16 (2013), pp. 7079-7090. ISSN: 0270-6474. DOI: 10.1523/JNEUROSCI .4286-12.2013. eprint: https : //www . jneurosci .org/content/33/16/ 7079.full.pdf. URL: https://www.jneurosci.org/content/33/16/7079.

[18] Alain-Sol Sznitman. "Topics in propagation of chaos". In: Ecole d'Ete de Probabilites de Saint-Flour XIX, vol.1464 (1989), pp. 165-251.

[19] Alexander A. Vladimirov, Serge A. Pirogov, Alexander N. Rybko, and Senya B. Shlosman. "Propagation of Chaos and Poisson Hypothesis". In: Problems of Information Transmission 54.3 (July 2018), 290-299. ISSN: 1608-3253. DOI: 10.1134/s0032946018030080. URL: http://dx.doi .org/ 10.1134/S0032946018030080. 